\title{
Weighted automata and weighted logics ${ }^{\star}$
}

\author{
Manfred Droste ${ }^{1}$ and Paul Gastin ${ }^{2}$ \\ 1 Institut für Informatik, Universität Leipzig \\ Augustusplatz 10-11, D-04109 Leipzig, Germany, \\ droste@informatik.uni-leipzig.de \\ 2 LSV, CNRS UMR 8643 \& ENS de Cachan \\ 61, Av. du Président Wilson, F-94235 Cachan Cedex, France, \\ Paul.Gastin@lsv.ens-cachan.fr
}

\begin{abstract}
Weighted automata are used to describe quantitative properties in various areas such as probabilistic systems, image compression, speech-to-text processing. The behaviour of such an automaton is a mapping, called a formal power series, assigning to each word a weight in some semiring. We generalize Büchi's and Elgot's fundamental theorems to this quantitative setting. We introduce a weighted version of MSO logic and prove that, for commutative semirings, the behaviours of weighted automata are precisely the formal power series definable with our weighted logic. We also consider weighted first-order logic and show that aperiodic series coincide with the first-order definable ones, if the semiring is locally finite, commutative and has some aperiodicity property.
\end{abstract}

\section{Introduction}

Büchi's and Elgot's fundamental theorems [3,9] established the coincidence of regular languages with languages definable in monadic second-order logic. At the same time, Schützenberger [22] investigated finite automata with weights and characterized their behaviours as rational formal power series. Both of these results have inspired a wealth of extensions and further research, cf. [24, 21, 15, 2] for surveys and monographs, and also led to recent practical applications, e.g. in verification of finite-state programs (model checking, $[17,1,16]$ ), in digital image compression $[5,11,13,12]$ and in speech-to-text processing $[19,20,4]$.

It is the goal of this paper to introduce a logic with weights and to show that the behaviours of weighted finite automata are precisely the series definable in our weighted monadic second-order logic. This can be viewed as a quantitative version of Büchi's and Elgot's classical (qualitative) results.

The syntax of our weighted logics incorporates weights taken from a semiring $K$, just as done for weighted automata in order to model a variety of applications and situations. The semantics of a weighted logic formula will be a formal power series with values in $K$. We restrict negation to atomic formulas since in general there is no natural complement operation in the semiring that would allow

\footnotetext{
* Work partly supported by the DAAD-PROCOPE project Temporal and Quantitative Analysis of Distributed Systems.
} 
us to define the semantics of negation elementwise. In comparison to classical MSO-logic, this is not a restriction, since we include disjunction, conjunction, existential and universal quantifications in our syntax. Thus, we obtain the classical MSO-logics by letting $K=\mathbb{B}$, the 2-element Boolean algebra.

Even for the semiring of natural numbers or the tropical semiring it turns out that neither universal first-order nor universal second-order quantification of formulas preserve recognizability. Therefore, for the syntax of restricted MSOlogic we exclude universal second-order quantification, and we permit universal first-order quantification only for formulas whose semantics takes finitely many values in $K$. Moreover, if we allow existential set quantifications only to occur at the beginning of a fomula, we arrive at restricted existential MSO-logic.

Now we give a summary of our results. First we show for any commutative semiring $K$ that the behaviours of weighted automata with values in $K$ are precisely the series definable by sentences of our restricted MSO-logic, or, equivalently, of our restricted existential MSO-logic. Second, if the semiring $K$ is locally finite, we obtain that the semantics of all sentences of our full weighted MSO-logic are representable by weighted automata. Locally finite semirings were investigated in [6]; they form a large class of semirings including e.g. all finite semirings, the max-min-semiring employed for capacity problems of networks, and all Boolean algebras. Thus we obtain Büchi's and Elgot's theorems as a particular consequence. Moreover, if the semiring $K$ is a field or locally finite and is given in some effective way, then the constructions in our proofs yield effective conversions of sentences of our weighted logics to weighted automata, and viceversa, and we obtain also decision procedures.

Finally, we investigate weighted first-order logic. As is well-known, the firstorder definable languages are precisely the starfree languages which in turn coincide with the the aperiodic ones $[23,18]$. Aperiodic and starfree formal power series were introduced and investigated in [6]. Easy examples show that even if the semiring $K$ is finite, series definable in our weighted first-order logic need not be aperiodic. However, we obtain that the aperiodic series coincide with the first-order definable ones, if the semiring is locally finite, commutative and both addition and multiplication satisfy a certain aperiodicity property. Such semirings include again all Boolean algebras, but also quite different ones like the truncated max-plus semiring.

We believe that the present paper opens a new research road. Obviously, one could try to extend our results to other structures such as trees or traces. One could also try to define weighted temporal logics and study not only expressiveness but also decidability and complexity of natural problems such as quantitative model checking.

Full proofs are available in the technical report [7].

\section{Weighted automata}

We give basic definitions and properties of semirings, formal power series and weighted automata. For background, we refer the reader to $[2,15,21]$. 
A semiring is a structure $(K,+, \cdot, 0,1)$ where $(K,+, 0)$ is a commutative monoid, $(K, \cdot, 1)$ is a monoid, multiplication distributes over addition, and $0 \cdot x=$ $x \cdot 0=0$ for each $x \in K$. If the multiplication is commutative, we say that $K$ is commutative. If the addition is idempotent, then the semiring is called idempotent. Important examples include

- the natural numbers $(\mathbb{N},+, \cdot, 0,1)$ with the usual addition and multiplication,

- the Boolean semiring $\mathbb{B}=(\{0,1\}, \vee, \wedge, 0,1)$,

- the tropical semiring Trop $=(\mathbb{N} \cup\{\infty\}$, min, $+, \infty, 0)$ (also known as minplus semiring), with min and + extended to $\mathbb{N} \cup\{\infty\}$ in the natural way,

- the arctical semiring $\operatorname{Arc}=(\mathbb{N} \cup\{-\infty\}$, max, $+,-\infty, 0)$,

- the semiring $([0,1]$, max $, \cdot, 0,1)$ which can be used to compute probabilities,

- the semiring of languages $\left(\mathcal{P}\left(A^{*}\right), \cup, \cap, \emptyset, A^{*}\right)$.

If $K$ is a semiring and $n \in \mathbb{N}$, then $K^{n \times n}$ comprises all $(n \times n)$-matrices over $K$. With usual matrix multiplication $\left(K^{n \times n}, \cdot\right)$ is a monoid.

A formal power series is a mapping $S: A^{*} \rightarrow K$. It is usual to write $(S, w)$ for $S(w)$. The set $\operatorname{Supp}(S):=\left\{w \in A^{*} \mid(S, w) \neq 0\right\}$ is called the support of $S$, and $\operatorname{Im}(S)=\left\{(S, w) \mid w \in A^{*}\right\}$ is the image of $S$. The set of all formal power series over $K$ and $A$ is denoted by $K\left\langle\left\langle A^{*}\right\rangle\right\rangle$. Now let $S, T \in K\left\langle\left\langle A^{*}\right\rangle\right\rangle$. The sum $S+T$ and the Hadamard product $S \odot T$ are both defined pointwise:

$(S+T, w):=(S, w)+(T, w)$ and $(S \odot T, w):=(S, w) \cdot(T, w) \quad\left(w \in A^{*}\right)$.

For $L \subseteq A^{*}$, we define the characteristic series $\mathbb{1}_{L}: A^{*} \rightarrow K$ by $\left(\mathbb{1}_{L}, w\right)=1$ if $w \in L$, and $\left(\mathbb{1}_{L}, w\right)=0$ otherwise.

Now we turn to weighted automata. We fix a semiring $K$ and an alphabet $A$. A weighted finite automaton over $K$ and $A$ is a quadruple $\mathcal{A}=(Q, \lambda, \mu, \gamma)$ where $Q$ is a finite set of states, $\mu: A \rightarrow K^{Q \times Q}$ is the transition weight function and $\lambda, \gamma: Q \rightarrow K$ are weight functions for entering and leaving a state, respectively. Here $\mu(a)$ is a $(Q \times Q)$-matrix whose $(p, q)$-entry $\mu(a)_{p, q} \in K$ indicates the weight of the transition $p \stackrel{a}{\longrightarrow} q$. Then $\mu$ extends uniquely to a monoid homomorphism (also denoted by $\mu$ ) from $A^{*}$ into $\left(K^{Q \times Q}, \cdot\right)$.

The weight of a path $P: q_{0} \stackrel{a_{1}}{\longrightarrow} q_{1} \longrightarrow \ldots \longrightarrow q_{n-1} \stackrel{a_{n}}{\longrightarrow} q_{n}$ in $\mathcal{A}$ is the product weight $(P):=\lambda\left(q_{0}\right) \cdot \mu\left(a_{1}\right)_{q_{0}, q_{1}} \cdots \mu\left(a_{n}\right)_{q_{n-1}, q_{n}} \cdot \gamma\left(q_{n}\right)$. This path has label $a_{1} \ldots a_{n}$. The weight of a word $w=a_{1} \ldots a_{n} \in A^{*}$ in $\mathcal{A}$, denoted $(\|\mathcal{A}\|, w)$, is the sum of weight $(P)$ over all paths $P$ with label $w$. One can check that $(\|\mathcal{A}\|, w)=\lambda \cdot \mu(w) \cdot \gamma$ with usual matrix multiplication, considering $\lambda$ as a row vector and $\gamma$ as a column vector. If $w=\varepsilon$, we have $(\|\mathcal{A}\|, \varepsilon)=\lambda \cdot \gamma$. This defines a formal power series $\|\mathcal{A}\|: A^{*} \rightarrow K$ called the behavior of $\mathcal{A}$. A formal power series $S \in K\left\langle\left\langle A^{*}\right\rangle\right\rangle$ is called recognizable, if there exists a weighted finite automaton $\mathcal{A}$ such that $S=\|\mathcal{A}\|$. Then we also call $\mathcal{A}$ or $(\lambda, \mu, \gamma)$ a representation of $S$. We let $K^{\text {rec }}\left\langle\left\langle A^{*}\right\rangle\right\rangle$ be the collection of all recognizable formal power series over $K$ and $A$.

Now let $h: A^{*} \rightarrow B^{*}$ be a homomorphism. If $T \in K\left\langle\left\langle B^{*}\right\rangle\right\rangle$, then $h^{-1}(T):=$ $T \circ h \in K\left\langle\left\langle A^{*}\right\rangle\right\rangle$. That is, $\left(h^{-1}(T), w\right)=(T, h(w))$ for each $w \in A^{*}$. We say that $h$ is non-erasing, if $h(a) \neq \varepsilon$ for any $a \in A$. In this case, for $S \in K\left\langle\left\langle A^{*}\right\rangle\right\rangle$, define 
$h(S): B^{*} \rightarrow K$ by $(h(S), v):=\sum_{w \in h^{-1}(v)}(S, w)\left(v \in B^{*}\right)$, noting that the sum is finite since $h$ is non-erasing. Recognizability is preserved by inverse morphisms and non-erasing morphisms $[8,2]$.

We say $S: A^{*} \rightarrow K$ is a recognizable step function, if $S=\sum_{i=1}^{n} k_{i} \cdot \mathbb{1}_{L_{i}}$ for some $n \in \mathbb{N}, k_{i} \in K$ and recognizable languages $L_{i} \subseteq A^{*}(i=1, \ldots, n)$. As is well-known, any recognizable step function is a recognizable power series.

\section{$3 \quad$ Weighted logics}

In this section, we introduce our weighted logics and study its first properties. We fix a semiring $K$ and an alphabet $A$. For each $a \in A, P_{a}$ denotes a unary predicate symbol. The syntax of formulas of the weighted MSO-logic is given by

$$
\begin{gathered}
\varphi::=k\left|P_{a}(x)\right| \neg P_{a}(x)|x \leq y| \neg(x \leq y)|x \in X| \neg(x \in X) \\
|\varphi \vee \psi| \varphi \wedge \psi|\exists x . \varphi| \exists X . \varphi|\forall x . \varphi| \forall X . \varphi
\end{gathered}
$$

where $k \in K$ and $a \in A$. We denote by $\operatorname{MSO}(K, A)$ the collection of all such weighted MSO-formulas $\varphi$.

Now we turn to the definition of the semantics of formulas $\varphi \in \operatorname{MSO}(K, A)$. We let Free $(\varphi)$ be the set of all free variables of $\varphi$. Let $w=a_{1} \ldots a_{n} \in A^{*}$ with $a_{i} \in A$. We also write $w(i)=a_{i}(1 \leq i \leq n)$. The length of $w$ is $|w|=n$.

Let $\mathcal{V}$ be a finite set of first-order and second-order variables. A $(\mathcal{V}, w)$ assignment $\sigma$ is a function mapping first-order variables in $\mathcal{V}$ to elements of $\{1, \ldots,|w|\}$ and second-order variables in $\mathcal{V}$ to subsets of $\{1, \ldots,|w|\}$. If $x$ is a first-order variable and $i \in\{1, \ldots,|w|\}$ then $\sigma[x \rightarrow i]$ is the $(\mathcal{V} \cup\{x\}, w)$ assignment which assigns $x$ to $i$ and acts like $\sigma$ on all other variables. Similarly, $\sigma[X \rightarrow I]$ is defined for $I \subseteq\{1, \ldots,|w|\}$.

As usual, a pair $(w, \sigma)$ where $\sigma$ is a $(\mathcal{V}, w)$-assignment will be encoded using an extended alphabet $A_{\mathcal{V}}=A \times\{0,1\}^{\mathcal{V}}$. More precisely, we will write a word over $A_{\mathcal{V}}$ as a pair $(w, \sigma)$ where $w$ is the projection over $A$ and $\sigma$ is the projection over $\{0,1\}^{\mathcal{V}}$. Now, $\sigma$ represents a valid assignment over $\mathcal{V}$ if for each first-order variable $x \in \mathcal{V}$, the $x$-row of $\sigma$ contains exactly one 1 . In this case, we identify $\sigma$ with the $(\mathcal{V}, w)$-assignment such that for each first-order variable $x \in \mathcal{V}, \sigma(x)$ is the position of the 1 on the $x$-row, and for each second-order variable $X \in \mathcal{V}$, $\sigma(X)$ is the set of positions carrying a 1 on the $X$-row. Clearly, the language $N_{\mathcal{V}}=\left\{(w, \sigma) \in A_{\mathcal{V}}^{*} \mid \sigma\right.$ is a valid $\mathcal{V}$-assignment $\}$ is recognizable. We simply write $A_{\varphi}=A_{\text {Free }(\varphi)}$ and $N_{\varphi}=N_{\text {Free }(\varphi)}$.

Definition 3.1. Let $\varphi \in \operatorname{MSO}(K, A)$ and $\mathcal{V}$ be a finite set of variables containing $\operatorname{Free}(\varphi)$. The semantics of $\varphi$ is a formal power series $\llbracket \varphi \rrbracket \mathcal{V} \in K\left\langle\left\langle A_{\mathcal{V}}^{*}\right\rangle\right\rangle$. Let $(w, \sigma) \in A_{\mathcal{V}}^{*}$. If $\sigma$ is not a valid $\mathcal{V}$-assignment, then we put $\llbracket \varphi \rrbracket \mathcal{V}(w, \sigma)=0$. Otherwise, we define $\llbracket \varphi \rrbracket \mathcal{V}(w, \sigma) \in K$ inductively as follows:

$$
\llbracket k \rrbracket \mathcal{V}(w, \sigma)=k
$$

$$
\llbracket P_{a}(x) \rrbracket \mathcal{V}(w, \sigma)= \begin{cases}1 & \text { if } w(\sigma(x))=a \\ 0 & \text { otherwise }\end{cases}
$$




$$
\begin{aligned}
\llbracket x \leq y \rrbracket \mathcal{V}(w, \sigma) & =\left\{\begin{array}{ll}
1 & \text { if } \sigma(x) \leq \sigma(y) \\
0 & \text { otherwise }
\end{array} \quad \llbracket x \in X \rrbracket \mathcal{V}(w, \sigma)= \begin{cases}1 & \text { if } \sigma(x) \in \sigma(X) \\
0 & \text { otherwise }\end{cases} \right. \\
\llbracket \neg \varphi \rrbracket \mathcal{V}(w, \sigma) & = \begin{cases}1 & \text { if } \llbracket \varphi \rrbracket \mathcal{V}(w, \sigma)=0 \quad \text { if } \varphi \text { is of the form } P_{a}(x),(x \leq y) \\
0 & \text { if } \llbracket \varphi \rrbracket \mathcal{V}(w, \sigma)=1 \quad \text { or }(x \in X) .\end{cases} \\
\llbracket \varphi \vee \psi \rrbracket \mathcal{V}(w, \sigma) & =\llbracket \varphi \rrbracket \mathcal{V}(w, \sigma)+\llbracket \psi \rrbracket \mathcal{V}(w, \sigma) \\
\llbracket \varphi \wedge \psi \rrbracket \mathcal{V}(w, \sigma) & =\llbracket \varphi \rrbracket \mathcal{V}(w, \sigma) \cdot \llbracket \psi \rrbracket \mathcal{V}(w, \sigma) \\
\llbracket \exists x . \varphi \rrbracket \mathcal{V}(w, \sigma) & =\sum_{1 \leq i \leq|w|}^{\llbracket \varphi \rrbracket \mathcal{V} \cup\{x\}}(w, \sigma[x \rightarrow i]) \\
\llbracket \exists X . \varphi \rrbracket \mathcal{V}(w, \sigma) & =\sum_{I \subseteq\{1, \ldots,|w|\}} \llbracket \varphi \rrbracket \mathbb{V} \cup\{X\}(w, \sigma[X \rightarrow I]) \\
\llbracket \forall x . \varphi \rrbracket \mathcal{V}(w, \sigma) & =\prod_{1 \leq i \leq|w|} \llbracket \varphi \rrbracket \mathcal{V} \cup\{x\} \\
\llbracket \forall X . \varphi \rrbracket \mathcal{V}(w, \sigma) & =\prod_{I \subseteq\{1, \ldots,|w|\}} \llbracket \varphi \rrbracket \mathbb{V} \cup\{X\}(w, \sigma[X \rightarrow i])
\end{aligned}
$$

where we fix some order on the power set of $\{1, \ldots,|w|\}$ so that the last product is defined even if $K$ is not commutative. We simply write $\llbracket \varphi \rrbracket$ for $\llbracket \varphi \rrbracket_{\operatorname{Free}(\varphi)}$.

Note that if $\varphi$ is a sentence, i.e. has no free variables, then $\llbracket \varphi \rrbracket \in K\left\langle\left\langle A^{*}\right\rangle\right\rangle$. We give several examples of possible interpretations for weighted formulas:

I. Let $K=(\mathbb{N},+, \cdot, 0,1)$ and assume $\varphi$ does not contain constants $k \in \mathbb{N}$. We may interpret $\llbracket \varphi \rrbracket(w, \sigma)$ as the number of proofs we have that $(w, \sigma)$ satisfies formula $\varphi$. Indeed, for atomic formulas the number of proofs is clearly 0 or 1 , depending on whether $\varphi$ holds for $(w, \sigma)$ or not. Now if e.g. $\llbracket \varphi \rrbracket(w, \sigma)=m$ and $\llbracket \psi \rrbracket(w, \sigma)=n$, the number of proofs that $(w, \sigma)$ satisfies $\varphi \vee \psi$ should be $m+n$ (since any proof suffices), and for $\varphi \wedge \psi$ it should be $m \cdot n$ (since we may pair the proofs of $\varphi$ and $\psi$ arbitrarily). Similarly, the semantics of the existential and universal quantifiers can be interpreted.

II. The formula $\exists x . P_{a}(x)$ counts how often $a$ occurs in the word. Here how often depends on the semiring: e.g. Boolean semiring, natural numbers, integers modulo $3, \ldots$

III. Consider the probability semiring $K=([0,1]$, $\max , \cdot, 0,1)$ and the alphabet $A=\left\{a_{1}, \ldots, a_{n}\right\}$. Assume that each letter $a_{i}$ has a reliability $k_{i}$. Then, the series assigning to a word its reliability can be given by the first-order formula $\forall x . \bigvee_{1 \leq i \leq n}\left(P_{a_{i}}(x) \wedge k_{i}\right)$.

IV. Let $K$ be an arbitrary Boolean algebra $\left(B, \vee, \wedge,{ }^{-}, 0,1\right)$. In this case, sums correspond to suprema, and products to infima. Here we can define the semantics of $\neg \varphi$ for an arbitrary formula $\varphi$ by $\llbracket \neg \varphi \rrbracket(w, \sigma):=\overline{\llbracket \varphi \rrbracket(w, \sigma)}$. Then clearly $\llbracket \varphi \wedge \psi \rrbracket=\llbracket \neg(\neg \varphi \vee \neg \psi) \rrbracket, \llbracket \forall x . \varphi \rrbracket=\llbracket \neg(\exists x . \neg \varphi) \rrbracket$ and $\llbracket \forall X . \varphi \rrbracket=$ $\llbracket \neg(\exists X . \neg \varphi) \rrbracket$. This may be interpreted as a multi-valued logics. In particular, if $K=\mathbb{B}$, the 2-valued Boolean algebra, our semantics coincides with the usual semantics of unweighted MSO-formulas, identifying characteristic series with their supports. 
Let $K$ be a semiring and $A$ an alphabet. Observe that if $\varphi \in \operatorname{MSO}(K, A)$, we have defined a semantics $\llbracket \varphi \rrbracket \mathcal{V}$ for each finite set of variables $\mathcal{V}$ containing $\operatorname{Free}(\varphi)$. Now we show that these semantics' are consistent with each other.

Proposition 3.2. Let $\varphi \in \operatorname{MSO}(K, A)$ and $\mathcal{V}$ a finite set of variables containing $\operatorname{Free}(\varphi)$. Then $\llbracket \varphi \rrbracket \mathcal{V}(w, \sigma)=\llbracket \varphi \rrbracket\left(w, \sigma_{\mid \operatorname{Free}(\varphi)}\right)$ for each $(w, \sigma) \in A_{\mathcal{V}}^{*}$ such that $\sigma$ is a valid $\mathcal{V}$-assignment. In particular, $\llbracket \varphi \rrbracket$ is recognizable iff $\llbracket \varphi \rrbracket \mathcal{V}$ is recognizable.

Proof (sketch). The first claim can be shown by induction on $\varphi$. For the final claim, let $\llbracket \varphi \rrbracket$ be recognizable, and consider the projection $\pi: A_{\mathcal{V}} \rightarrow A_{\varphi}$. For $(w, \sigma) \in A_{\mathcal{V}}^{*}$, we have $\pi(w, \sigma)=\left(w, \sigma_{\mid \operatorname{Free}(\varphi)}\right)$. Hence, $\llbracket \varphi \rrbracket \mathcal{V}=\pi^{-1}(\llbracket \varphi \rrbracket) \odot \mathbb{1}_{N_{\mathcal{V}}}$ is recognizable by standard preservation results, cf. [2]. The converse can be shown similarly.

Now let $Z \subseteq \operatorname{MSO}(K, A)$. A series $S: A^{*} \rightarrow K$ is called $Z$-definable, if there is a sentence $\varphi \in Z$ such that $S=\llbracket \varphi \rrbracket$. The main goal of this paper is the comparison of $Z$-definable with recognizable series, for suitable fragments $Z$ of $\operatorname{MSO}(K, A)$. Crucial for this will be closure properties of recognizable series under the constructs of our weighted logic. However, first we will show that $K^{\mathrm{rec}}\left\langle\left\langle A^{*}\right\rangle\right\rangle$ is in general not closed under universal quantification.

Example 3.3. Let $K=(\mathbb{N},+, \cdot, 0,1)$. Then $\llbracket \forall x .2 \rrbracket(w)=2^{|w|}$ and $\llbracket \forall y \forall x .2 \rrbracket(w)=$ $\left(2^{|w|}\right)^{|w|}=2^{|w|^{2}}$. Clearly, the series $\llbracket \forall x .2 \rrbracket$ is recognizable by the weighted automaton $(Q, \lambda, \mu, \gamma)$ with $Q=\{1\}, \lambda_{1}=\gamma_{1}=1$ and $\mu_{1,1}(a)=2$ for all $a \in A$. However, $\llbracket \forall y \forall x .2 \rrbracket$ is not recognizable. Suppose there was an automaton $\mathcal{A}^{\prime}=\left(Q^{\prime}, \lambda^{\prime}, \mu^{\prime}, \gamma^{\prime}\right)$ with behavior $\llbracket \forall y \forall x .2 \rrbracket$. Let $M=\max \left\{\left|\lambda_{p}^{\prime}\right|,\left|\gamma_{p}^{\prime}\right|,\left|\mu^{\prime}(a)_{p, q}\right| \mid\right.$ $\left.p, q \in Q^{\prime}, a \in A\right\}$. Then $\left(\left\|\mathcal{A}^{\prime}\right\|, w\right) \leq\left|Q^{\prime}\right|^{|w|+1} \cdot M^{|w|+2}$ for any $w \in A^{*}$, a contradiction with $\left(\left\|\mathcal{A}^{\prime}\right\|, w\right)=2^{|w|^{2}}$.

A similar argument applies also for the tropical and the arctical semirings. Observe that in all these cases, $\llbracket \forall x .2 \rrbracket$ has infinite image.

Example 3.4. Let $K=(\mathbb{N},+, \cdot, 0,1)$. Then $\llbracket \forall X .2 \rrbracket(w)=2^{2^{|w|}}$ for any $w \in A^{*}$, and as above $\llbracket \forall X .2 \rrbracket$ is not recognizable due to its growth. Again, this counterexample also works for the tropical and the arctical semirings.

The examples show that unrestricted universal quantification is too strong to preserve recognizability. This motivates the following definition. We will call a formula $\varphi \in \operatorname{MSO}(K, A)$ restricted, if it contains no universal set quantification of the form $\forall X . \psi$, and whenever $\varphi$ contains a universal first-order quantification $\forall x . \psi$, then $\llbracket \psi \rrbracket$ is a recognizable step function. We let $\operatorname{RMSO}(K, A)$ comprise all restricted formulas of $\operatorname{MSO}(K, A)$. Furthermore, let $\operatorname{REMSO}(K, A)$ contain all restricted existential MSO-formulas $\varphi$, i.e. $\varphi$ is of the form $\varphi=\exists X_{1}, \ldots, X_{n} . \psi$ with $\psi \in \operatorname{RMSO}(K, A)$ containing no set quantification.

We let $K^{\mathrm{rmso}}\left\langle\left\langle A^{*}\right\rangle\right\rangle$ (resp. $\left.K^{\mathrm{remso}}\left\langle\left\langle A^{*}\right\rangle\right\rangle\right)$ contain all series $S \in K\left\langle\left\langle A^{*}\right\rangle\right\rangle$ which are definable by some sentence in $\operatorname{RMSO}(K, A)$ (resp. in $\operatorname{RemSO}(K, A))$. The main result of this paper is the following theorem. It will be proved in sections 4 and 5 . 
Theorem 3.5. Let $K$ be a commutative semiring and $A$ an alphabet. Then

$$
K^{\mathrm{rec}}\left\langle\left\langle A^{*}\right\rangle\right\rangle=K^{\mathrm{rmso}}\left\langle\left\langle A^{*}\right\rangle\right\rangle=K^{\mathrm{remso}}\left\langle\left\langle A^{*}\right\rangle\right\rangle .
$$

\section{Definable series are recognizable}

In all of this section, let $K$ be a semiring and $A$ an alphabet. We wish to show that if $K$ is commutative, then all RMSO-definable series $\llbracket \varphi \rrbracket$ over $K$ and $A$ are recognizable. We proceed by induction over the structure of RMSO-formulas.

Lemma 4.1. Let $\varphi, \psi \in \operatorname{MSO}(K, A)$.

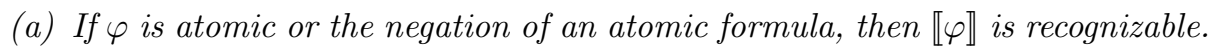

(b) If $\llbracket \varphi \rrbracket$ and $\llbracket \psi \rrbracket$ are recognizable, then $\llbracket \varphi \vee \psi \rrbracket$ is recognizable. If in addition $K$ is commutative, then $\llbracket \varphi \wedge \psi \rrbracket$ is also recognizable.

(c) If $\llbracket \varphi \rrbracket$ is recognizable, then $\llbracket \exists x . \varphi \rrbracket$ and $\llbracket \exists X . \varphi \rrbracket$ are recognizable series.

Proof (sketch). (a) Automata with behavior $\llbracket \varphi \rrbracket$ can easily be given explicitly.

(b) Let $\mathcal{V}=\operatorname{Free}(\varphi) \cup \operatorname{Free}(\psi)$. By definition, we have $\llbracket \varphi \vee \psi \rrbracket=\llbracket \varphi \rrbracket \mathcal{V}+\llbracket \psi \rrbracket \mathcal{V}$ and $\llbracket \varphi \wedge \psi \rrbracket=\llbracket \varphi \rrbracket \mathcal{V} \odot \llbracket \psi \rrbracket_{\mathcal{V}}$. Hence the result follows from Proposition 3.2 and since sum and Hadamard product preserve recognizability of series $[8,2]$.

(c) Let $\mathcal{V}=\operatorname{Free}(\exists X . \varphi)$ and consider the projection $\pi: A_{\mathcal{V} \cup\{X\}}^{*} \rightarrow A_{\mathcal{V}}^{*}$. We can show that $\llbracket \exists X . \varphi \rrbracket(w, \sigma)=\pi\left(\llbracket \varphi \rrbracket_{\mathcal{V} \cup\{X\}}\right)(w, \sigma)$. Now, $\llbracket \varphi \rrbracket_{\mathcal{V} \cup\{X\}}$ is recognizable by Proposition 3.2 and since non-erasing projections preserve recognizability we get $\llbracket \exists X . \varphi \rrbracket$ recognizable. The case $\exists x . \varphi$ can be dealt with similarly.

The most interesting and new case here arises from universal quantification.

Lemma 4.2. Let $K$ be commutative and $\varphi \in \operatorname{MSO}(K, A)$ such that $\llbracket \varphi \rrbracket$ is a recognizable step function. Then $\llbracket \forall x . \varphi \rrbracket$ is recognizable.

Proof. Let $\mathcal{W}=\operatorname{Free}(\varphi)$ and $\mathcal{V}=\operatorname{Free}(\forall x . \varphi)=\mathcal{W} \backslash\{x\}$. We may write $\llbracket \varphi \rrbracket=$ $\sum_{j=1, \ldots, n} k_{j} \cdot \mathbb{1}_{L_{j}}$ with $n \in \mathbb{N}, k_{j} \in K$ and recognizable languages $L_{j} \subseteq A_{\mathcal{W}}^{*}$ $(j=1, \ldots, n)$ forming a partition of $A_{\mathcal{W}}^{*}$.

First, we assume that $x \in \mathcal{W}$. Let $\widetilde{A}=A \times\{1, \ldots, n\}$. A word in $(\widetilde{A} \mathcal{V})^{*}$ will be written $(w, \nu, \sigma)$ where $(w, \sigma) \in A_{\mathcal{V}}^{*}$ and $\nu \in\{1, \ldots, n\}^{*}$ is interpreted as a mapping from $\{1, \ldots,|w|\}$ to $\{1, \ldots, n\}$. Let $\widetilde{L}$ be the set of $(w, \nu, \sigma) \in$ $\left(\widetilde{A}_{\mathcal{V}}\right)^{*}$ such that for all $i \in\{1, \ldots,|w|\}$ and $j \in\{1, \ldots, n\}$ we have $\nu(i)=$ $j$ implies $(w, \sigma[x \rightarrow i]) \in L_{j}$. Observe that for each $(w, \sigma) \in A_{\mathcal{V}}^{*}$ there is a unique $\nu$ such that $(w, \nu, \sigma) \in \widetilde{L}$ since the $L_{j}$ form a partition of $A_{\mathcal{W}}^{*}$.

It can be shown that $\widetilde{L}$ is a recognizable word language. Hence there is a deterministic automaton $\widetilde{\mathcal{A}}$, with state set $Q$, say, recognizing $\widetilde{L}$. Now we obtain a weighted automaton $\mathcal{A}$ with the same state set by adding weights to the transitions of $\widetilde{\mathcal{A}}$ as follows: If $(p,(a, j, s), q)$ is a transition in $\widetilde{\mathcal{A}}$ with $p, q \in Q$, $(a, s) \in A_{\mathcal{V}}$ and $1 \leq j \leq n$, we let this transition in $\mathcal{A}$ have weight $k_{j}$, i.e. $\mu_{\mathcal{A}}(a, j, s)_{p, q}=k_{j}$. All triples which are not transitions in $\widetilde{\mathcal{A}}$ get weight 0 . Also, 
the initial state of $\widetilde{\mathcal{A}}$ gets initial weight 1 in $\mathcal{A}$, all non-initial states of $\widetilde{\mathcal{A}}$ get initial weight 0 , and similarly for the final states and final weights.

Clearly, since $\widetilde{\mathcal{A}}$ is deterministic and accepts $\widetilde{L}$, the weight of $(w, \nu, \sigma) \in \widetilde{L}$ in $\mathcal{A}$ is $\prod_{1 \leq j \leq n} k_{j}^{\left|\nu^{-1}(j)\right|}$, and the weight of $(w, \nu, \sigma) \in \widetilde{A}^{*} \backslash \widetilde{L}$ in $\mathcal{A}$ is 0 . Now let $h:\left(\widetilde{A}_{\mathcal{V}}\right)^{*} \rightarrow A_{\mathcal{V}}^{*}$ be the projection mapping $(w, \nu, \sigma)$ to $(w, \sigma)$. Then for any $(w, \sigma) \in A_{\mathcal{V}}^{*}$ and the unique $\nu$ such that $(w, \nu, \sigma) \in \widetilde{L}$ we obtain

$$
h(\|\mathcal{A}\|)(w, \sigma)=\sum_{\rho}\|\mathcal{A}\|(w, \rho, \sigma)=\|\mathcal{A}\|(w, \nu, \sigma)=\prod_{1 \leq j \leq n} k_{j}^{\left|\nu^{-1}(j)\right|} .
$$

Now we have

$$
\llbracket \forall x . \varphi \rrbracket(w, \sigma)=\prod_{1 \leq i \leq|w|} \llbracket \varphi \rrbracket(w, \sigma[x \rightarrow i])=\prod_{1 \leq j \leq n} k_{j}^{\left|\nu^{-1}(j)\right|}
$$

where the last equality holds due to the form of $\varphi$. Hence $\llbracket \forall x . \varphi \rrbracket=h(\|\mathcal{A}\|)$ which is recognizable $[8,2]$.

Now assume that $x \notin \mathcal{W}$, so that $\mathcal{V}=\mathcal{W}$. Let $\varphi^{\prime}=\varphi \wedge(x \leq x)$. So $\llbracket \varphi^{\prime} \rrbracket$ is recognizable by Lemma 4.1, and clearly $\llbracket \varphi \rrbracket_{\mathcal{V} \cup\{x\}}=\llbracket \varphi^{\prime} \rrbracket \mathcal{V} \cup\{x\}$. Thus $\llbracket \forall x . \varphi \rrbracket \mathcal{V}=$ $\llbracket \forall x . \varphi^{\prime} \rrbracket \mathcal{V}$ which is recognizable by what we showed above.

Now the following result is immediate by Lemmata 4.1 and 4.2.

Theorem 4.3. Let $K$ be a commutative semiring, $A$ an alphabet and $\varphi \in$ $\operatorname{RMSO}(K, A)$. Then $\llbracket \varphi \rrbracket \in K^{\mathrm{rec}}\left\langle\left\langle A^{*}\right\rangle\right\rangle$ is recognizable.

Next we turn to decidability questions. Employing decidability results from the theory of formal power series and our previous constructions, we can show:

Proposition 4.4. Let $K$ be a computable field, and let $\varphi \in \operatorname{MSO}(K, A)$. It is decidable whether $\varphi$ is restricted, and in this case one can effectively compute a weighted automaton $\mathcal{A}_{\varphi}$ for $\llbracket \varphi \rrbracket$.

Corollary 4.5. Let $K$ be a computable field, and let $\varphi, \psi \in \operatorname{RMSO}(K, A)$. Then it is decidable whether $\llbracket \varphi \rrbracket=\llbracket \psi \rrbracket$. It is also decidable whether $\llbracket \varphi \rrbracket$ and $\llbracket \psi \rrbracket$ differ only for finitely many words.

Proof. By Proposition 4.4, the series $\llbracket \varphi \rrbracket, \llbracket \psi \rrbracket$ and hence also $\llbracket \varphi \rrbracket-\llbracket \psi \rrbracket=\llbracket \varphi \rrbracket+$ $(-1) \cdot \llbracket \psi \rrbracket$ are effectively recognizable. By [2, Propositions VI.1.1, VI.1.2], it is decidable whether such a series equals 0 , or whether its support is finite.

\section{Recognizable series are definable}

In all of this section let $K$ be a semiring and $A$ an alphabet. We wish to show that if $K$ is commutative, then all recognizable series are REMSO-definable. For this, the concept of an unambiguous MSO-formula will be useful. The class of unambiguous formulas in $\operatorname{MSO}(K, A)$ is defined inductively as follows: All 
atomic formulas of the form $P_{a}(x), x \leq y$ or $(x \in X)$, and their negations are unambiguous. If $\varphi, \psi$ are unambiguous, then $\varphi \wedge \psi, \forall x . \varphi$ and $\forall X$. $\varphi$ are also unambiguous. If $\varphi, \psi$ are unambiguous and $\operatorname{Supp}(\llbracket \varphi \rrbracket) \cap \operatorname{Supp}(\llbracket \psi \rrbracket)=\emptyset$, then $\varphi \vee \psi$ is unambiguous. Let $\varphi$ be unambiguous and $\mathcal{V}=\operatorname{Free}(\varphi)$. If for any $(w, \sigma) \in A_{\mathcal{V}}^{*}$ there is at most one element $i \in\{1, \ldots,|w|\}$ such that $\llbracket \varphi \rrbracket_{\mathcal{V} \cup\{x\}}(w, \sigma[x \rightarrow i]) \neq 0$, then $\exists x . \varphi$ is unambiguous. If for any $(w, \sigma) \in A_{\mathcal{V}}^{*}$ there is at most one subset $I \subseteq$ $\{1, \ldots,|w|\}$ such that $\llbracket \varphi \rrbracket \mathcal{V} \cup\{X\}(w, \sigma[X \rightarrow I]) \neq 0$, then $\exists X . \varphi$ is unambiguous.

Proposition 5.1. Let $\varphi \in \operatorname{MSO}(K, A)$ be unambiguous. We may also regard $\varphi$ as a classical MSO-formula defining the language $\mathcal{L}(\varphi) \subseteq A_{\varphi}^{*}$. Then, $\llbracket \varphi \rrbracket=\mathbb{1}_{\mathcal{L}(\varphi)}$ is a recognizable step function.

Next we show that, conversely, classical MSO-formulas can be transformed into unambiguous formulas.

Lemma 5.2. For each classical MSO-formula $\varphi$ not containing set quantifications (but possibly including atomic formulas of the form $(x \in X)$ ) we can effectively construct two unambiguous $\operatorname{MSO}(K, A)$-formula $\varphi^{+}$and $\varphi^{-}$such that $\llbracket \varphi^{+} \rrbracket=\mathbb{1}_{\mathcal{L}(\varphi)}$ and $\llbracket \varphi^{-} \rrbracket=\mathbb{1}_{\mathcal{L}(\neg \varphi)}$.

Proof (sketch). We may assume (using also conjunction and universal quantification in our syntax or as abbreviations) that in $\varphi$ negations are applied only to atomic formulas. Now we proceed by induction, giving only the formulas for some cases: $(\varphi \vee \psi)^{-}=\varphi^{-} \wedge \psi^{-},(\varphi \vee \psi)^{+}=\varphi^{+} \vee\left(\varphi^{-} \wedge \psi^{+}\right),(\exists x . \varphi)^{-}=\forall x . \varphi^{-}$ and $(\exists x \cdot \varphi)^{+}=\exists x \cdot\left(\varphi^{+}(x) \wedge \forall y \cdot\left((x \leq y) \vee\left(\neg(x \leq y) \wedge \varphi^{-}(y)\right)\right)\right)$.

Theorem 5.3. Let $K$ be commutative. Then $K^{\mathrm{rec}}\left\langle\left\langle A^{*}\right\rangle\right\rangle \subseteq K^{\mathrm{remso}}\left\langle\left\langle A^{*}\right\rangle\right\rangle$.

Proof (sketch). Let $\mathcal{A}=(Q, \lambda, \mu, \gamma)$ be a weighted automaton over $A$. For each triple $(p, a, q) \in Q \times A \times Q$ choose a set variable $X_{p, a, q}$, and let $\mathcal{V}=\left\{X_{p, a, q} \mid\right.$ $p, q \in Q, a \in A\}$. We choose an enumeration $X_{1}, \ldots, X_{m}$ of $\mathcal{V}$ with $m=|Q|^{2} \cdot|A|$. In the formulas below we use classical macros such as partition $\left(X_{1}, \ldots, X_{m}\right)$, $(y=x+1), \min (y)$ and $\max (y)$. Define the unambiguous formula

$$
\begin{aligned}
\psi\left(X_{1}, \ldots, X_{m}\right) & :=\operatorname{partition}\left(X_{1}, \ldots, X_{m}\right)^{+} \wedge \bigwedge_{p, a, q} \forall x \cdot\left(\left(x \in X_{p, a, q}\right) \rightarrow P_{a}(x)\right)^{+} \\
& \wedge \forall x \forall y \cdot\left((y=x+1) \underset{p, q, r \in Q, a, b \in A}{\rightarrow} \bigvee_{p, a, q}\left(x \in X_{p, a}\right) \wedge\left(y \in X_{q, b, r}\right)\right)^{+} .
\end{aligned}
$$

Let $w=a_{1} \ldots a_{n} \in A^{+}$. One can show that there is a bijection between the set of paths in $\mathcal{A}$ over $w$ and the set of $(w, \mathcal{V})$-assignments $\sigma$ satisfying $\psi$. Consider now the formula $\varphi\left(X_{1}, \ldots, X_{m}\right)$ defined by

$$
\begin{aligned}
& \psi\left(X_{1}, \ldots, X_{m}\right) \wedge\left(\bigwedge_{p, a, q} \forall x \cdot\left(\neg\left(x \in X_{p, a, q}\right) \vee\left(\left(x \in X_{p, a, q}\right) \wedge \mu(a)_{p, q}\right)\right)\right) \wedge \\
& \quad \exists y .\left(\min (y) \wedge \bigvee_{p, a, q}\left(y \in X_{p, a, q}\right) \wedge \lambda_{p}\right) \wedge \exists z \cdot\left(\max (z) \wedge \bigvee_{p, a, q}\left(z \in X_{p, a, q}\right) \wedge \gamma_{q}\right) .
\end{aligned}
$$


Let $\rho$ be a path in $\mathcal{A}$ over $w$ and let $\sigma_{\rho}$ be the associated $(w, \mathcal{V})$-assignment. We can show that $\llbracket \varphi \rrbracket \mathcal{V}\left(w, \sigma_{\rho}\right)=$ weight $(\rho)$. Let $\xi=\exists X_{1} \cdots \exists X_{m} \cdot \varphi\left(X_{1}, \ldots, X_{m}\right)$. For $w \in A^{+}$we can show that $(\llbracket \xi \rrbracket, w)=(\|\mathcal{A}\|, w)$. Let $\zeta=(\lambda \cdot \gamma) \wedge \forall x . \neg(x \leq x)$. Then, $\|\mathcal{A}\|=\llbracket \zeta \vee \xi \rrbracket \in K^{\mathrm{remso}}\left\langle\left\langle A^{*}\right\rangle\right\rangle$.

Now Theorem 3.5 is immediate by Theorems 4.3 and 5.3 .

Observe that the proof of Theorem 5.3 is constructive, i.e. given a weighted automaton $\mathcal{A}$, we effectively obtain an $\operatorname{REMSO}(K, A)$-sentence $\varphi$ with $\llbracket \varphi \rrbracket=$ $\|\mathcal{A}\|$. Using this, from the theory of formal power series (cf. $[21,15,2]$ ) we immediately obtain undecidablilty results for the semantics of weighted MSOsentences. For instance, it is undecidable whether a given REMSO-sentence $\varphi$ over $\mathbb{Q}$, the field of rational numbers, and an alphabet $A$, satisfies $\operatorname{Supp}(\llbracket \varphi \rrbracket)=$ $A^{*}$. Also, by a result of Krob [14], the equality of given recognizable series over the tropical semiring is undecidable. Hence, the equality of two given REMSO(Trop, $A$ )-sentences is also undecidable.

\section{Locally finite semirings}

In section 3 we gave examples of semirings $K$ showing that the results of Theorem 3.5 and 4.3 in general do not hold for arbitrary $\operatorname{MSO}(K, A)$-sentences. In contrast, here we wish to show that for a large class of semirings $K$, all $\operatorname{MSO}(K, A)$-formulas have a recognizable semantics.

A semiring $K$ is called locally finite, if each finitely generated subsemiring of $K$ is finite. For example, any Boolean algebra $(B, \vee, \wedge, 0,1)$ is locally finite. The max-min semiring $\mathbb{R}_{\max , \min }=\left(\mathbb{R}_{+} \cup\{\infty\}\right.$, max, min, $\left.0, \infty\right)$ of positive reals, used in operations research for maximum capacity problems of networks, is locally finite. In fact, more generally, any distributive lattice $(L, \vee, \wedge, 0,1)$ with smallest element 0 and largest element 1 is a locally finite semiring, cf. [6] for further basic properties. Examples of infinite but locally finite fields are provided by the algebraic closures of the finite fields $\mathbf{Z} / p \mathbf{Z}$ for any prime $p$. We can show (see [7]):

Theorem 6.1. Let $K$ be a locally finite commutative semiring and $A$ an alphabet. Then $K^{r e c}\left\langle\left\langle A^{*}\right\rangle\right\rangle=K^{m s o}\left\langle\left\langle A^{*}\right\rangle\right\rangle$.

Again, given an $\operatorname{MSO}(K, A)$-formula $\varphi$, we can effectively construct a weighted automaton $\mathcal{A}$ over $K$ and $A_{\varphi}$ such that $\|\mathcal{A}\|=\llbracket \varphi \rrbracket$. As a consequence of this and of corresponding decidability results given in the full version of [6] for recognizable series over locally finite semirings, we immediately obtain:

Corollary 6.2. Let $K$ be a locally finite commutative semiring and $A$ an alphabet. It is decidable whether two given $\operatorname{MSO}(K, A)$-formulas $\varphi$ and $\psi$ satisfy $\llbracket \varphi \rrbracket=\llbracket \psi \rrbracket ;$ whether a given $\operatorname{MSO}(K, A)$-formula $\varphi$ satisfies $\operatorname{Supp}(\llbracket \varphi \rrbracket)=A_{\varphi}^{*}$.

\section{Weighted first-order logic}

In this section, we investigate weighted first-order logic and the relationship to aperiodic series. Most of our results will require additional assumptions on the semiring $K$. 
Let $K$ be a semiring and $A$ an alphabet. A formula $\varphi \in \operatorname{MSO}(K, A)$ is called a (weighted) first-order formula, if $\varphi$ does not contain any set variable. We let $\operatorname{FO}(K, A)$ contain all first-order formulas and $\operatorname{RFO}(K, A)$ all restricted first-order formulas over $K$ and $A$. The collections of series definable by these formulas are denoted $K^{f o}\left\langle\left\langle A^{*}\right\rangle\right\rangle$ and $K^{r f o}\left\langle\left\langle A^{*}\right\rangle\right\rangle$, respectively.

As is well-known, the first-order definable languages are precisely the starfree languages which in turn coincide with the the aperiodic ones [23, 18]. Aperiodic and starfree formal power series were introduced and investigated in [6]. Recall that a monoid $M$ is said to be aperiodic, if there exists some $m \geq 0$ such that $x^{m}=x^{m+1}$ for all $x \in M$. We call a monoid $M$ weakly aperiodic, if for each $x \in M$ there exists $m \geq 0$ such that $x^{m}=x^{m+1}$. Clearly, a finite monoid is aperiodic iff it is weakly aperiodic.

A series $S: A^{*} \rightarrow K$ is called aperiodic, if there exists a representation $S=$ $(Q, \lambda, \mu, \gamma)$ with $\mu\left(A^{*}\right)$ aperiodic. Observe that then there exists some $m \geq 0$ such that for all $w \in A^{*}$ we have $\mu\left(w^{m}\right)=\mu\left(w^{m+1}\right)$ and hence $\left(S, w^{m}\right)=\left(S, w^{m+1}\right)$. The collection of all aperiodic series over $K$ and $A$ will be denoted $K^{\text {aper }}\left\langle\left\langle\mathcal{A}^{*}\right\rangle\right\rangle$.

Now we turn to the relationship between aperiodic and FO-definable series. First we show that even if $K$ is finite and commutative, in general we do not have $K^{\text {aper }}\left\langle\left\langle A^{*}\right\rangle\right\rangle=K^{\text {fo }}\left\langle\left\langle A^{*}\right\rangle\right\rangle$.

Example 7.1. Let $K=\mathbb{Z} / 2 \mathbb{Z}$, the field with two elements, and $S=\llbracket \exists x .1 \rrbracket$. Then $S(w)=|w| \bmod 2$ for any $w \in A^{*}$. Hence $S$ is not aperiodic since otherwise we would obtain some $m \geq 1$ such that $S\left(a^{m}\right)=S\left(a^{m+1}\right)(a \in A)$, a contradiction. Note that here the monoid $(K, \cdot)$ is idempotent, and $(K,+)$ is not aperiodic.

Example 7.2. Let $K$ be the tropical semiring and $T=\llbracket \forall x .1 \rrbracket$. Then $T(w)=|w|$ for all $w \in A^{*}$, so $T$ is not aperiodic. Note that $(\mathbb{N} \cup\{-\infty\}, \max )$ is idempotent, but $(\mathbb{N} \cup\{-\infty\},+)$ is not weakly aperiodic.

These examples indicate that in order to achieve the inclusion $K^{f o}\left\langle\left\langle A^{*}\right\rangle\right\rangle \subseteq$ $K^{\text {aper }}\left\langle\left\langle A^{*}\right\rangle\right\rangle$, we need some aperiodicity assumption both for $(K,+)$ and $(K, \cdot)$.

We call a semiring $K$ weakly bi-aperiodic, if both $(K,+)$ and $(K, \cdot)$ are weakly aperiodic. If $K$ is also commutative, then in particular $K$ is locally finite. Clearly, any idempotent monoid is weakly aperiodic. Thus the weakly bi-aperiodic semirings include all semirings in which both addition and multiplication are idempotent, and this class of semirings properly contains (cf. [10]) the class of all distributive lattices $(L, \vee, \wedge, 0,1)$ with smallest element 0 and greatest element 1. There are further examples:

Example 7.3. Let $0<d \in \mathbb{R}$. We let $\mathbb{R}_{\max }^{d}$ be the real max - plus semiring truncated at $d$, i.e. $\mathbb{R}_{\max }^{d}=\left([0, d] \cup\{-\infty\}, \max ,+_{d},-\infty, 0\right)$ with $x+{ }_{d} y:=x+y$ if $x+y \leq d$, and $x+{ }_{d} y:=d$ if $x+y \geq d$. This semiring is weakly bi-aperiodic, and $\left(\mathbb{R}_{\max }^{d},+_{d}\right)$ is weakly aperiodic but not aperiodic.

We can show (see [7]):

Theorem 7.4. Let $K$ be a commutative weakly bi-aperiodic semiring, and $A$ an alphabet. Then $K^{\text {aper }}\left\langle\left\langle A^{*}\right\rangle\right\rangle=K^{f o}\left\langle\left\langle A^{*}\right\rangle\right\rangle=K^{r f o}\left\langle\left\langle A^{*}\right\rangle\right\rangle$. 


\section{References}

1. A. Arnold. Finite Transition Systems. International Series in Computer Science. Prentice Hall, 1994.

2. J. Berstel and Ch. Reutenauer. Rational Series and Their Languages, volume 12 of EATCS Monographs in Theoretical Computer Science. Springer Verlag, 1988.

3. J.R. Büchi. Weak second-order arithmetic and finite automata. Z. Math. Logik Grundlagen Math., 6:66-92, 1960.

4. A.L. Buchsbaum, R. Giancarlo, and J.R. Westbrook. On the determinization of weighted finite automata. SIAM Journal on Computing, 30(5):1502-1531, 2000.

5. K. Culik and J. Kari. Image compression using weighted finite automata. Computer and Graphics, 17:305-313, 1993.

6. M. Droste and P. Gastin. On aperiodic and star-free formal power series in partially commuting variables. In Proccedings of FPSAC'00, pages 158-169. Springer, 2000. Full version available as a Research Report, LSV, ENS de Cachan, France, 2005.

7. M. Droste and P. Gastin. Weighted automata and weighted logics. Research Report LSV-05-02, ENS de Cachan, France, 2005. 23 pages.

8. S. Eilenberg. Automata, Languages and Machines, volume A. Academic Press, New York, 1974.

9. C.C. Elgot. Decision problems of finite automata design and related arithmetics. Trans. Amer. Math. Soc., 98:21-52, 1961.

10. S. Golan. Semirings and their Applications. Kluwer Academic Publisher, 1999.

11. U. Hafner. Low Bit-Rate Image and Video Coding with Weighted Finite Automata. $\mathrm{PhD}$ thesis, Universität Würzburg, Germany, 1999.

12. Z. Jiang, B. Litow, and O. de Vel. Similarity enrichment in image compression through weighted finite automata. In $C O C O O N^{\prime} 00$, number 1858 in Lecture Notes in Computer Science, pages 447-456. Springer Verlag, 2000.

13. F. Katritzke. Refinements of data compression using weighted finite automata. $\mathrm{PhD}$ thesis, Universität Siegen, Germany, 2001.

14. D. Krob. The equality problem for rational series with multiplicities in the tropical semiring is undecidable. International Journal of Algebra and Computation, 4(3):405-425, 1994.

15. W. Kuich and A. Salomaa. Semirings, Automata, Languages, volume 6 of EATCS Monographs in Theoretical Computer Science. Springer Verlag, 1986.

16. R.P. Kurshan. Computer-Aided Verification of Coordinating Processes. Princeton Series in Computer Science. Princeton University Press, 1994.

17. K. McMillan. Symbolic Model Checking. Kluwer Academic Publishers, 1993.

18. R. McNaughton and S. Papert. Counter-free automata. MIT Press, Cambridge, 1971.

19. M. Mohri. Finite-state transducers in language and speech processing. Computational Linguistics, 23:269-311, 1997.

20. M. Mohri, F. Pereira, and M. Riley. The design principles of a weighted finite-state transducer library. Theoretical Computer Science, 231:17-32, 2000.

21. A. Salomaa and M. Soittola. Automata-Theoretic Aspects of Formal Power Series. Texts and Monographs in Computer Science. Springer Verlag, 1978.

22. M.P. Schützenberger. On the definition of a family of automata. Information and Control, 4:245-270, 1961.

23. M.P. Schützenberger. On finite monoids having only trivial subgroups. Information and Control, 8:190-194, 1965.

24. W. Thomas. Languages, automata and logic. In G. Rozenberg and A. Salomaa, editors, Handbook of Formal Languages, Vol. 3, pages 389-485. Springer Verlag, 1997. 